\title{
Quantum probes of timelike naked singularities in the weak field regime of $f(R)$ global monopole spacetime
}

\author{
O. Gurtug, M. Halilsoy and S. Habib Mazharimousavi \\ Department of Physics, Eastern Mediterranean University, \\ G. Magusa, north Cyprus, Mersin 10, Turkey \\ E-mail: ozay.gurtug@emu.edu.tr, mustafa.halilsoy@emu.edu.tr, \\ habib.mazhari@emu.edu.tr
}

ABSTRACT: The formation of a naked singularity in $f(R)$ global monopole spacetime is considered in view of quantum mechanics. Quantum test fields obeying the Klein-Gordon, Dirac and Maxwell equations are used to probe the classical timelike naked singularity developed at $r=0$. We prove that the spatial derivative operator of the fields fails to be essentially self-adjoint. As a result, the classical timelike naked singularity formed in $f(R)$ global monopole spacetime remains quantum mechanically singular when it is probed with quantum fields having different spin structures. Pitelli and Letelier (Phys. Rev. D 80 (2009) 104035) had shown that for quantum scalar (spin 0) probes the general relativistic global monopole singularity remains intact. For specific modes electromagnetic (spin 1) and Dirac field (spin 1/2) probes, however, we show that the global monopole spacetime behaves quantum mechanically regular. The admissibility of this singularity is also incorporated within the Gubser's singularity conjecture.

Keywords: Classical Theories of Gravity, Spacetime Singularities

ARXIV EPRINT: 1312.4453 


\section{Contents}

1 Introduction $\quad 2$

2 The metric for a global monopole in $f(R)$ theories and spacetime structure

2.1 The metric for a global monopole in $f(R)$ theories

2.2 The spacetime structure

2.2.1 Case 1: when $\alpha^{2}>8 \psi_{0} G M$.

2.2.2 Case 2: when $\alpha^{2}=8 \psi_{0} G M$.

2.2.3 Case 3: when $\alpha^{2}<8 \psi_{0} G M$.

2.3 The description of the $f(R)$ global monopole spacetime in a Newman-Penrose (NP) formalism

4 Quantum singularities in $f(R)$ global monopole spacetime

4.1 Klein-Gordon fields

4.1.1 The case of $\mathrm{r} \rightarrow \infty$

4.1.2 The case of $\mathrm{r} \rightarrow 0$

4.2 Maxwell fields

$\begin{array}{lll}\text { 4.2.1 } & \text { For the case } r \rightarrow \infty & 15\end{array}$

$\begin{array}{lll}\text { 4.2.2 The case } \mathrm{r} \rightarrow 0 & 15\end{array}$

4.3 Dirac fields 16

$\begin{array}{lll}\text { 4.3.1 For the case } r \rightarrow \infty & 18\end{array}$

$\begin{array}{lll}\text { 4.3.2 For the case } \mathrm{r} \rightarrow 0 & 18\end{array}$

5 Probing the singularity around BV spacetime with Maxwell and Dirac fields

5.1 Maxwell fields 20

5.1.1 For the case $\mathrm{r} \rightarrow \infty \quad 20$

5.1.2 For the case $\mathrm{r} \rightarrow 0 \quad 20$

5.2 Dirac fields 21

5.2.1 For the case $\mathrm{r} \rightarrow \infty \quad 21$

5.2.2 For the case $\mathrm{r} \rightarrow 0 \quad 22$

6 Analysis with Gubser's singularity conjecture 22

$\begin{array}{llr}7 & \text { Conclusion } & 23\end{array}$ 


\section{Introduction}

Spacetime singularities are believed to be one of the inevitable consequences of the Einstein's theory of relativity. It describes the "end point" or incomplete geodesics for timelike or null trajectories followed by classical particles. The black hole and colliding plane wave spacetimes are the two important branches of this theory that the nature and characteristics of spacetime singularities are manifested. Another intriguing one is the Big-Bang-like cosmological singularities. According to the classical singularity classification devised by Ellis and Schmidt [1], curvature singularities can be grouped as scalar and nonscalar. The scalar curvature singularities are the strongest ones in the sense that the spacetime becomes inextendible and all the physical quantities, such as the gravitational field, energy density and tidal forces, diverge at the singular point. Singularities forming at the centre of black holes and in some colliding plane wave spacetimes are good examples for strong scalar curvature singularity. In black hole spacetimes singularities located at the centre $(r=0)$ is hidden by horizon(s). In the cases where this singularity is not hidden, it is called the naked singularity. Whereas, the singularity occurring in the interaction region of Bell-Szekeres solution [2] which describes the nonlinear interaction of electromagnetic plane waves can be given as an example to nonscalar curvature singularity.

Naked singularity which is visible from outside needs further care as far as the weak cosmic censorship hypothesis is concerned. It is believed that, naked singularity forms a threat to this hypothesis. Hence, understanding and the resolution of naked singularities seems to be extremely important for the deterministic nature of general relativity.

However, the scale where the singularities are forming is very small (smaller than the Planck scale), so that the classical general relativity methods in the resolution of the singularities are expected to be replaced by the quantum theory of gravity. Unfortunately, there is no consistent quantum theory of gravity yet. Since this theory is still "under construction", the alternative methods in healing the singularities are always attracted the attentions. String theory [3,4] and loop quantum gravity [5] constitute two major study fields in resolving singularities. It is shown in string theory that some timelike singularities are resolved: the orbifold, the flop, and the conifold. The flop and the conifold occurs in the Calabi-Yau manifolds in which their resolution involves the use of light matters such as "twisted sectors" and "wrapped D-branes" [6] (and references therein).

A rather different approach is considered in [7] for resolving the timelike singularities in Reissner-Nordström and negative mass Schwarzschild solutions. In this approach, the spacetime is viewed as being made of two parts which are naturally connected across the singularity. In this study, it is shown that the Reissner-Nordström singularity allows for communication through the singularity and can be termed as "beam splitter" since the transmission probability of a suitably prepared high energy wave packet is $25 \%$.

Another alternative method; following the work of Wald [8], is proposed by Horowitz and Marolf (HM) [9], which incorporates "self-adjointness" of the spatial part of the wave operator. Hence, the classical notion of geodesics incompleteness with respect to pointparticle probe will be replaced by the notion of quantum singularity with respect to wave probes. 
The method of HM has been used successfully for other spacetimes to check whether the classically singular spacetimes are quantum mechanically regular or not. As an example; negative mass Schwarzschild spacetime, charged dilatonic black hole spacetime and fundamental string spacetimes are considered in [9]. An alternative function space, namely the Sobolev space instead of the Hilbert space, has been introduced in [10], for analyzing the singularities within the framework of quantum mechanics. As a result, the occurrence of timelike naked singularity in the negative mass Schwarzschild solution is shown to be quantum mechanically regular. Helliwell and Konkowski have studied quasiregular [11], Gal'tsovLetelier-Tod spacetime [12], Levi-Civita spacetimes [13, 14], and recently, they have also considered conformally static spacetimes $[15,16]$. Pitelli and Letelier have studied spherical and cylindrical topological defects [17], Banados-Teitelboim-Zanelli (BTZ) spacetimes [18], the global monopole spacetime [19] and cosmological spacetimes [20]. Quantum singularities in matter coupled $2+1$ dimensional black hole spacetimes are considered in [21]. Quantum singularities are also considered in Lovelock theory [22] and linear dilaton black hole spacetimes [23]. The occurrence of naked singularities in a $2+1$ dimensional magnetically charged solution in Einstein-Power-Maxwell theory have also been considered [24]. Recently, the formation of naked singularity in a model of $f(R)$ gravity is considered in [25].

The main motivation in these studies is to understand whether these classically singular spacetimes turn out to be quantum mechanically regular if they are probed with quantum fields rather than classical particles.

Recently, a solution describing $f(R)$ global monopole in the weak field regime has been presented in [26]. This study showed that, the main contribution of the modified theory compared to the ordinary global monopole solution due to Barriola and Vilenkin (BV) [27] is that, in addition to admitting double and single horizons, it admits solution without horizon as well. And, the most important influence is seen on the nature of the singularity that occur at $r=0$. In the case of $\mathrm{BV}$, this singularity is spacelike, whereas in the case of $f(R)$ theory, it has timelike nature.

Generally, solutions admitting black holes attracted more attention than the solutions admitting naked singularity. Recently, the influence of the modified theory on the thermodynamic quantities of an $f(R)$ global monopole spacetime [26] has been investigated and compared with BV spacetime in [28]. The outcome of this investigation is that, $f(R)$ theory modifies the thermodynamic quantities, but the shapes of curves for thermodynamic quantities with respect to the horizon are similar to the results within the frame of general relativity.

In this paper, we wish to investigate the occurrence of timelike naked singularities in $f(R)$ global monopole spacetime within the context of quantum mechanics. The singularity at $r=0$ will be probed with three different types of quantum fields that obey Klein-Gordon, Maxwell and Dirac equations. The singularity for the BV spacetime will also be investigated with the spinor fields obeying Maxwell and Dirac equations. This will be the spinor field generalization of the study performed by Pitelli and Letelier [19] for BV spacetime.

The appearance of naked singularities are also encountered in gauged supergravity theories. Gubser [29] proposed a singularity conjecture to resolve singularities in these theories in the following way. 
Conjecture: large curvatures in scalar coupled gravity with four dimensional Poincare invariant solution are allowed only if the scalar potential is bounded above in the solution.

In this paper, the approach of Gubser will be incorporated to our analysis briefly to display its applicability in spacetimes which do not obey Poincare invariance.

The paper is organized as follows: in section II, we give the solution and the spacetime structure obtained in [26]. The definition of quantum singularity is briefly reviewed in section III. Section IV is devoted for the quantum singularity analysis of the $f(R)$ global monopole spacetime. Three different types of waves with different spins are used to probe the singularity. The spinor field generalization of the paper by Pitelli and Letelier [19] is given in section V. In section VI, Gubser's singularity conjecture is used to identify if the studied curvature singularity is bad or good. Finally, we give the concluding remarks of this study in section VII.

\section{The metric for a global monopole in $f(R)$ theories and spacetime struc- ture}

\subsection{The metric for a global monopole in $f(R)$ theories}

Recently, the metric describing the global monopole in $f(R)$ theories for the static spherically symmetric systems has been presented in the weak field regime [26]. The adopted action for such a gravitational field coupled to matter fields in $f(R)$ theory is given by

$$
S=\frac{1}{2 \kappa} \int d^{4} x \sqrt{-g} f(R)+S_{m},
$$

in which $f(R)$ is an analytic function of the Ricci scalar $R, \kappa=8 \pi G$, here $G$ is the Newton constant and $S_{m}$ represents the action of the coupled matter fields given by

$$
S_{m}=\int d^{4} x \sqrt{-g} \mathcal{L} .
$$

In the considered global monopole model, $\mathcal{L}$ represents the Lagrangian density that gives the simplest global monopole model given by

$$
\mathcal{L}=\frac{1}{2} \partial_{\mu} \phi^{a} \partial^{\mu} \phi^{a}-\frac{1}{4} \lambda\left(\phi^{a} \phi^{a}-\eta^{2}\right)
$$

in which $\lambda$ and $\eta$ are constant parameters. The global monopole, that forms as a result of spontaneous symmetry breaking from global $O(3)$ to $\mathrm{U}(1)$, during the phase transitions in the early universe is described by the self-coupling triplet of scalar fields $\phi^{a}(a=1,2,3)$ given by the following ansatz,

$$
\phi^{a}=\eta \frac{x^{a}}{r},
$$

with $x^{a} x^{a}=r^{2}$ and $\eta$ is a constant parameter. The adopted metric for such a model is given by

$$
d s^{2}=B d t^{2}-A d r^{2}-r^{2}\left(d \theta^{2}+\sin ^{2} \theta d \varphi^{2}\right)
$$


where $B=B(r)$ and $A=A(r)$ are only function of $r$. The field equation reads

$$
F(R) R_{\mu}^{\nu}+\left(\square F(R)-\frac{1}{2} f(R)\right) \delta_{\mu}^{\nu}-\nabla^{\nu} \nabla_{\mu} F(R)=\kappa T_{\mu}^{\nu}
$$

in which

$$
\begin{aligned}
F(R) & =\frac{d f(R)}{d R}, \\
\square F(R) & =\frac{1}{\sqrt{-g}} \partial_{\mu}\left(\sqrt{-g} \partial^{\mu}\right) F(R)
\end{aligned}
$$

and

$$
\nabla^{\nu} \nabla_{\mu} F(R)=g^{\alpha \nu}\left[(F(R))_{, \mu, \alpha}-\Gamma_{\mu \alpha}^{m}(F(R))_{, m}\right] .
$$

In eq. (2.6) $T_{\mu}^{\nu}$ represents minimally coupled energy - momentum tensor of the matter field whose non-zero components are given by

$$
T_{0}^{0}=T_{r}^{r}=-\frac{8 \pi G \eta^{2}+3 G M \psi_{0}}{r^{2}}+\frac{3-16 \pi G \eta^{2}}{r} \psi_{0}+3 \psi_{0}^{2} .
$$

Furthermore, the trace of the field equation (2.6) reads

$$
F(R) R+3 \square F(R)-2 f(R)=\kappa T,
$$

with $T=T_{\mu}^{\mu}$. With reference to the paper [26], the solution to the field equations was obtained in the weak field regime which assumes the metric function in the form of $B=1+b(r)$ and $A=1+a(r)$ with the property that $|a(r)|$ and $|b(r)|$ smaller than unity. As a consequence of a weak field regime, the considered model of $f(R)$ theory corresponds to a small correction on standard general relativity in such a way that, $F(R(r))=1+\psi(r)$ with $\psi(r) \ll 1$. Explicit form of $f(R)$ is given in [26] (eq. 42 in [26]). Hence, $F(R(r))=1$ corresponds to the standard general relativity. Employing these conditions in the field equations yields $\psi(r)=\psi_{0} r$ and resulting metric function with global monopole is found to be

$$
B=A^{-1}=1-8 \pi G \eta^{2}-\frac{2 G M}{r}-\psi_{0} r
$$

where $M$ is the mass parameter and $\psi_{0}$ is a very small parameter ( since $\psi_{0} r \ll 1$ ) that measures the deviation from the standard general relativity. As stated in [26], for a typical Grand Unified Theory the parameter $\eta$ is in the order of $10^{16} \mathrm{GeV}$. Hence, $8 \pi G \eta^{2} \approx 10^{-5}$. Note that one can recover the result of BV if $\psi_{0}=0$. It is known that, the global monopole solution obtained by BV has one horizon only and the nature of the singularity at $r=0$ is spacelike.

\subsection{The spacetime structure}

The structure of the solution obtained in [26] and given in eq. (2.12), has remarkable features that deserves to be investigated in detail. The obtained solution admits black 
holes with inner and outer horizons. To find the location of the horizon, we prefer to write the metric component $g_{t t}$ in the following form

$$
B=-\frac{\psi_{0}}{r}\left(r-r_{+}\right)\left(r-r_{-}\right)
$$

where $r_{+}$and $r_{-}$denote the outer and inner horizons respectively and given by

$$
r_{ \pm}=\frac{\alpha \pm \sqrt{\alpha^{2}-8 \psi_{0} G M}}{2 \psi_{0}}, \quad \alpha=1-8 \pi G \eta^{2}
$$

The Kretschmann scalar which indicates the formation of curvature singularity for the $f(R)$ global monopole is given by

$$
\mathcal{K}=\frac{4}{r^{6}}\left\{2 \psi_{0}^{2} r^{4}+\left(16 \psi_{0} \pi G \eta^{2}\right) r^{3}+\left(8 \pi G \eta^{2}\right)^{2} r^{2}+\left(32 \pi G^{2} M \eta^{2}\right) r+12 G M^{2}\right\} .
$$

It is evident that $r=0$ is a typical central curvature singularity that is peculiar to the spherically symmetric systems. In order to find the nature or the character of the singularity at $r=0$ for the $f(R)$ global monopole, we perform conformal compactification. The conformal radial or tortoise coordinate is given by

$$
r_{*}=\int \frac{d r}{B}=-\frac{1}{\psi_{0}\left(r_{+}-r_{-}\right)}\left\{r_{+} \ln \left|r-r_{+}\right|-r_{-} \ln \left|r-r_{-}\right|\right\} .
$$

The retarded and advanced coordinates are defined as $u=t-r_{*}$ and $v=t+r_{*}$ respectively. Defining the Kruskal coordinates as

$$
\begin{aligned}
& u^{\prime}=\exp \left(\frac{\psi_{0}\left(r_{+}-r_{-}\right)}{2 r_{-}} u\right), \\
& v^{\prime}=\exp \left(-\frac{\psi_{0}\left(r_{+}-r_{-}\right)}{2 r_{-}} v\right),
\end{aligned}
$$

the metric can be written as

$$
d s^{2}=\frac{4 r_{-}^{2}\left(r-r_{+}\right)^{\frac{r_{+}+r_{-}}{r_{-}}}}{\psi_{0} r\left(r_{+}-r_{-}\right)^{2}} d u^{\prime} d v^{\prime}-r^{2}\left(d \theta^{2}+\sin ^{2} \theta d \varphi^{2}\right),
$$

and

$$
u^{\prime} v^{\prime}=\left(r-r_{-}\right)\left(r-r_{+}\right)^{-r_{+} / r_{-}}
$$

In order to bring infinity into a finite coordinate, we define

$$
\begin{array}{ll}
u^{\prime \prime}=\arctan u^{\prime}, & 0<u^{\prime \prime}<\pi / 2, \\
v^{\prime \prime}=\arctan v^{\prime}, & 0<v^{\prime \prime}<\pi / 2 .
\end{array}
$$

The corresponding Carter-Penrose diagrams for the following three possible cases are plotted and given in figures. The singularity located at $r=0$ is shown vertically on the Carter-Penrose diagram which indicates timelike character.

There are three possible cases to be investigated. 


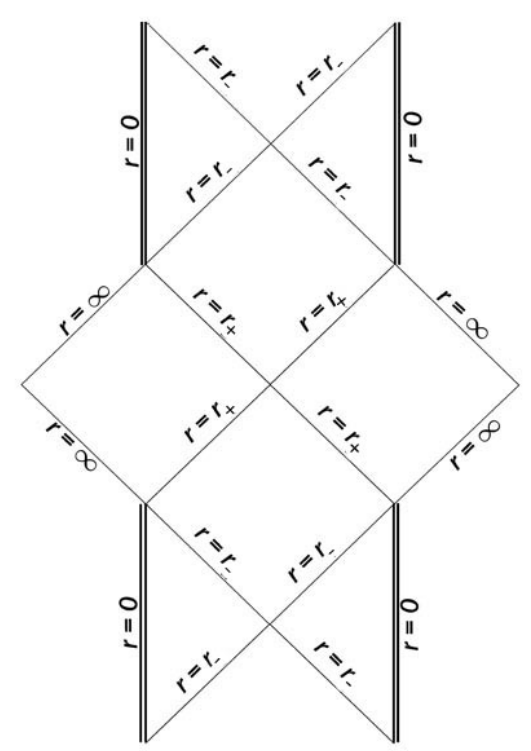

Figure 1. Carter-Penrose diagram of the $f(R)$ global monopole spacetime with inner $r_{-}$and outer $r_{+}$horizons. Timelike singularity is at $r=0$.

\subsubsection{Case 1: when $\alpha^{2}>8 \psi_{0} G M$}

The metric function, $B(r)=\alpha-\frac{2 G M}{r}-\psi_{0} r$, admits two positive roots $r_{+}$and $r_{-}$, indicating the location of the outer and inner horizons of a black hole. The Penrose diagram for this case is shown in figure 1 .

\subsubsection{Case 2: when $\alpha^{2}=8 \psi_{0} G M$}

The metric function, $B(r)=\alpha-\frac{2 G M}{r}-\psi_{0} r$, admits one horizon only. It can be interpreted as the extreme black hole. The Penrose diagram of this case is given in figure 2. Recently, the thermodynamic properties of the black hole solutions of $f(R)$ global monopole is investigated and presented in [27].

\subsubsection{Case 3: when $\alpha^{2}<8 \psi_{0} G M$}

In this case, the metric function, $B(r)=\alpha-\frac{2 G M}{r}-\psi_{0} r$, does not admit real roots. Hence, the solution in this particular case is not a black hole solution and the singularity at $r=0$ becomes timelike naked singularity, as depicted in the Penrose diagram in figure 3 . The choice of the parameters of the $f(R)$ global monopole metric results with timelike naked singularity at $r=0$ or black hole solutions with one or two horizons. These results seem to show that the small correction to the standard general relativity produces significant changes on the spacetime structure of the BV metric obtained by Barriola and Vilenkin.

In this paper, we are aiming to investigate this singularity within the context of quantum mechanics. This classically singular spacetime will be probed with quantum waves obeying the Klein-Gordon, Maxwell and Dirac equations to check whether the timelike naked singularity is smoothed out or not. 


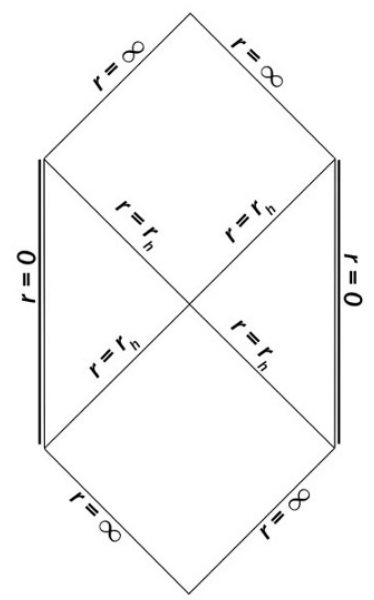

Figure 2. Carter-Penrose diagram of $f(R)$ global monopole spacetime with a single horizon at $r=r_{h}$

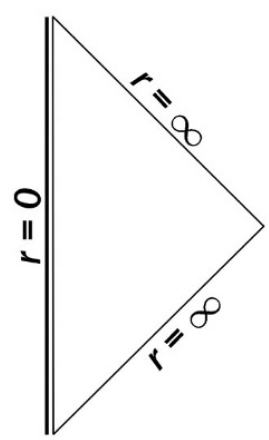

Figure 3. Carter-Penrose diagram for $f(R)$ global monopole spacetime without a horizon in which $r=0$ is a naked timelike singularity.

\subsection{The description of the $f(R)$ global monopole spacetime in a Newman- Penrose (NP) formalism}

The $f(R)$ global monopole metric is investigated with the Newman-Penrose (NP) formalism, in order to clarify the contribution of the $f(R)$ gravity. The set of proper null tetrads $1-$ forms is given by

$$
\begin{aligned}
l & =d t-\frac{d r}{B(r)}, \\
n & =\frac{1}{2}(B(r) d t+d r), \\
m & =-\frac{r}{\sqrt{2}}(d \theta+i \sin \theta d \varphi) . \\
\bar{m} & =-\frac{r}{\sqrt{2}}(d \theta-i \sin \theta d \varphi)
\end{aligned}
$$


The non-zero spin coefficients in these tetrads are

$$
\begin{array}{ll}
\beta=-\alpha=\frac{\cot \theta}{2 \sqrt{2} r}, & \rho=-\frac{1}{r}, \\
\mu=-\frac{B}{2 r}, & \gamma=\frac{1}{4} \frac{d B}{d r} .
\end{array}
$$

As a result, we obtain the Weyl and the Ricci scalars as

$$
\begin{aligned}
\Psi_{2} & =-\frac{3 G M+4 \pi G \eta^{2} r}{3 r^{3}}, \\
\phi_{11} & =\frac{8 \pi G \eta^{2}+\psi_{0} r}{4 r^{2}}, \\
\Lambda & =\frac{8 \pi G \eta^{2}+3 \psi_{0} r}{12 r^{2}},
\end{aligned}
$$

so that the spacetime is Petrov type $-D$. The parameter $\psi_{0}$ representing the contribution of $f(R)$ gravity is seen to effect only the Ricci components, leaving the mass term $\Psi_{2}$ of an ordinary global monopole unchanged.

\section{Quantum singularities}

Horowitz and Marolf (HM) [9], by developing the pioneering work of Wald [8], have proposed a prescription which involves the use of quantum particles/waves to judge whether the classical timelike curvature singularities occurring in static spacetimes are smoothed out quantum mechanically or not. According to HM, the singular character of the spacetime is defined as the ambiguity in the evolution of the wave functions. That is to say, the singular character is determined in terms of the ambiguity when attempting to find a self-adjoint extension of the spatial part of the wave operator to the entire Hilbert space. If the extension is unique, it is said that the space is quantum mechanically regular. A brief review now follows: consider a static spacetime $\left(M, g_{\mu \nu}\right)$ with a timelike Killing vector field $\xi^{\mu}$. Let $t$ denote the Killing parameter and $\Sigma$ denote a static slice. The Klein-Gordon equation in this space is

$$
\left(\nabla^{\mu} \nabla_{\mu}-m^{2}\right) \psi=0 .
$$

This equation can be written in the form

$$
\frac{\partial^{2} \psi}{\partial t^{2}}=\sqrt{f} D^{i}\left(\sqrt{f} D_{i} \psi\right)-f m^{2} \psi=-A \psi
$$

in which $f=-\xi^{\mu} \xi_{\mu}$ and $D_{i}$ is the spatial covariant derivative on $\Sigma$. The Hilbert space $\mathcal{H},\left(L^{2}(\Sigma)\right)$ is the space of square integrable functions on $\Sigma$. The domain of an operator $A, D(A)$, is taken in such a way that it does not enclose the spacetime singularities. An appropriate set is $C_{0}^{\infty}(\Sigma)$, the set of smooth functions with compact support on $\Sigma$. The operator $A$ is real, positive and symmetric; therefore, its self-adjoint extensions always exist. If it has a unique extension $A_{E}$, then $A$ is called essentially self-adjoint [30-32]. Accordingly, the Klein-Gordon equation for a free particle satisfies

$$
i \frac{d \psi}{d t}=\sqrt{A_{E}} \psi
$$


with the solution

$$
\psi(t)=\exp \left[-i t \sqrt{A_{E}}\right] \psi(0)
$$

If $A$ is not essentially self-adjoint, the future time evolution of the wave function (3.4) is ambiguous. Then the HM criterion defines the spacetime as quantum mechanically singular. However, if there is only a single self-adjoint extension, the operator $A$ is said to be essentially self-adjoint and the quantum evolution described by eq. (3.4) is uniquely determined by the initial conditions. According to the HM criterion, this spacetime is said to be quantum mechanically non-singular. In order to determine the number of self-adjoint extensions, the concept of deficiency indices is used. The deficiency subspaces $N_{ \pm}$are defined by (see ref. [10] for a detailed mathematical background)

$$
\begin{aligned}
& N_{+}=\left\{\psi \in D\left(A^{*}\right), A^{*} \psi=Z_{+} \psi, \operatorname{Im} Z_{+}>0\right\} \\
& \quad \text { with dimension } n_{+} \\
& N_{-}=\left\{\psi \in D\left(A^{*}\right), A^{*} \psi=Z_{-} \psi, \operatorname{Im} Z_{-}<0\right\} \\
& \quad \text { with dimension } n_{-}
\end{aligned}
$$

The dimensions $\left(n_{+}, n_{-}\right)$are the deficiency indices of the operator $A$. The indices $n_{+}\left(n_{-}\right)$ are completely independent of the choice of $Z_{+}\left(Z_{-}\right)$depending only on whether or not $Z$ lies in the upper (lower) half complex plane. Generally one takes $Z_{+}=i \lambda$ and $Z_{-}=-i \lambda$, where $\lambda$ is an arbitrary positive constant necessary for dimensional reasons. The determination of deficiency indices is then reduced to counting the number of solutions of $A^{*} \psi=Z \psi$; (for $\lambda=1$ ),

$$
A^{*} \psi \pm i \psi=0
$$

that belong to the Hilbert space $\mathcal{H}$. If there are no square integrable solutions (i.e. $n_{+}=n_{-}=0$ ), the operator $A$ possesses a unique self-adjoint extension and is essentially self-adjoint. Consequently, the way to find a sufficient condition for the operator $A$ to be essentially self-adjoint is to investigate the solutions satisfying eq. (3.7) that do not belong to the Hilbert space.

\section{Quantum singularities in $f(R)$ global monopole spacetime}

\subsection{Klein-Gordon fields}

The massive Klein-Gordon equation for a scalar particle with mass $m$ can be written as

$$
\left(g^{-1 / 2} \partial_{\mu}\left[g^{1 / 2} g^{\mu \nu} \partial_{\nu}\right]-m^{2}\right) \psi=0
$$

For the metric (2.5), the Klein-Gordon equation can be splitted into a time and spatial part and written as

$$
\frac{\partial^{2} \psi}{\partial t^{2}}=-B\left\{B \frac{\partial^{2} \psi}{\partial r^{2}}+\frac{1}{r^{2}} \frac{\partial^{2} \psi}{\partial \theta^{2}}+\frac{1}{r^{2} \sin ^{2} \theta} \frac{\partial^{2} \psi}{\partial \varphi^{2}}+\frac{\cot \theta}{r^{2}} \frac{\partial \psi}{\partial \theta}+\left(\frac{2 B}{r}+B^{\prime}\right) \frac{\partial \psi}{\partial r}\right\}+B m^{2} \psi
$$


In analogy with eq. (3.2), the spatial operator $A$ for the massless case is

$$
A=B\left\{B \frac{\partial^{2}}{\partial r^{2}}+\frac{1}{r^{2}} \frac{\partial^{2}}{\partial \theta^{2}}+\frac{1}{r^{2} \sin ^{2} \theta} \frac{\partial^{2}}{\partial \varphi^{2}}+\frac{\cot \theta}{r^{2}} \frac{\partial}{\partial \theta}+\left(\frac{2 B}{r}+B^{\prime}\right) \frac{\partial}{\partial r}\right\},
$$

and the equation to be solved is $\left(A^{*} \pm i\right) \psi=0$.Using separation of variables, $\psi=$ $R(r) Y_{l}^{m}(\theta, \varphi)$, we get the radial part of eq. (3.7) as

$$
R^{\prime \prime}+\frac{\left(r^{2} B\right)^{\prime}}{r^{2} B} R^{\prime}+\left(\frac{-l(l+1)}{r^{2} B} \pm \frac{i}{B^{2}}\right) R=0,
$$

whose solutions represents spin 0 bosonic waves and a prime denotes the derivative with respect to $r$. The spatial operator $A$ is esentially self adjoint if neither of two solutions of eq. (4.4) is square integrable over all space $L^{2}(0, \infty)$. Because of the complexity in finding exact analytic solution to eq. (4.4), we study the behavior of $R(r)$ near $r \rightarrow \infty$ and $r \rightarrow 0$.

\subsubsection{The case of $\mathrm{r} \rightarrow \infty$}

The case $r \rightarrow \infty$ is topologically different compared to the analysis for ordinary global monopole solutions reported in [19]. The asymptotic behavior of the $f(R)$ global monopole metric when $r \rightarrow \infty$ is not conical and given by

$$
d s^{2} \simeq-\left(\alpha-\psi_{0} r\right) d t^{2}+\frac{d r^{2}}{\left(\alpha-\psi_{0} r\right)}+r^{2}\left(d \theta^{2}+\sin ^{2} \theta d \varphi^{2}\right) .
$$

For the above metric, the radial equation (4.4), for $r \rightarrow \infty$ becomes,

$$
R^{\prime \prime} \pm \frac{i}{\left(\alpha-\psi_{0} r\right)} R=0
$$

whose solution is

$R_{ \pm}=C_{1} \sqrt{\alpha-\psi_{0} r} J_{1}\left[( \pm 1+i) \sqrt{2} \sqrt{\frac{\alpha-\psi_{0} r}{\psi_{0}^{2}}}\right]+C_{2} \sqrt{\alpha-\psi_{0} r} N_{1}\left[( \pm 1+i) \sqrt{2} \sqrt{\frac{\alpha-\psi_{0} r}{\psi_{0}^{2}}}\right]$

where $C_{1}$ and $C_{2}$ are arbitrary integration constants, $J_{1}$ and $N_{1}$ are the first and second kind Bessel functions. The square integrability of the above solution for each sign \pm is checked by calculating the squared norm of the above solution in which the function space on each $t=$ constant hypersurface $\Sigma$ is defined as $\mathcal{H}=\{R \mid\|R\|<\infty\}$. The squared norm for the metric (4.5) is given by,

$$
\|R\|^{2}=\int_{r}^{\infty} \frac{\left|R_{ \pm}(r)\right|^{2} r^{2}}{\left(\alpha-\psi_{0} r\right)} d r .
$$

Our calculation has revealed that the obtained solution at infinity fails to satisfy square integrability condition i.e. $\|R\|^{2} \rightarrow \infty$. Hence, the solution at infinity does not belong to the Hilbert space. 


\subsubsection{The case of $\mathrm{r} \rightarrow 0$}

The approximate metric near the origin is Schwarzschild like and given by

$$
d s^{2} \simeq-\left(\alpha-\frac{2 G M}{r}\right) d t^{2}+\frac{d r^{2}}{\left(\alpha-\frac{2 G M}{r}\right)}+r^{2}\left(d \theta^{2}+\sin ^{2} \theta d \varphi^{2}\right) .
$$

The radial equation (4.4), for the above metric reduces to

$$
R^{\prime \prime}-\frac{\beta}{r} R=0,
$$

in which $\beta=\frac{l(l+1)}{2 G M}$, and the solution is obtained in terms of first and second kind of Bessel's functions and given by

$$
R=C_{3} \sqrt{r} J_{1}(2 \sqrt{\beta r})+C_{4} \sqrt{r} N_{1}(2 \sqrt{\beta r})
$$

where $C_{3}$ and $C_{4}$ are arbitrary integration constants. The square integrability of the above solution is checked by calculating the squared norm for the metric (4.9) which is given by,

$$
\|R\|^{2}=\int_{0}^{\text {constant }} \frac{|R|^{2} r^{2}}{\left(\alpha-\frac{2 G M}{r}\right)} d r<\infty
$$

which is always square integrable near $r=0$. Consequently, the spatial operator $A$ is not square integrable over all space $L^{2}(0, \infty)$ and therefore, it is not essentially self-adjoint. Hence, the classical singularity at $r=0$ remains quantum mechanically singular when probed with fields obeying the Klein-Gordon equation.

In the next subsections, the singularity will be probed with spinorial fields obeying Maxwell and Dirac equations. We prefer to use same method and terminology reported in $[25]$.

\subsection{Maxwell fields}

The Newman-Penrose formalism will be used to find the source-free Maxwell fields propagating in the space of $f(R)$ global monopole spacetime. The four coupled source-free Maxwell equations for electromagnetic fields in the Newman-Penrose formalism is given by

$$
\begin{aligned}
D \phi_{1}-\bar{\delta} \phi_{0} & =(\pi-2 \alpha) \phi_{0}+2 \rho \phi_{1}-\kappa \phi_{2}, \\
\delta \phi_{2}-\Delta \phi_{1} & =-\nu \phi_{0}+2 \mu \phi_{1}+(\tau-2 \beta) \phi_{2}, \\
\delta \phi_{1}-\Delta \phi_{0} & =(\mu-2 \gamma) \phi_{0}+2 \tau \phi_{1}-\sigma \phi_{2}, \\
D \phi_{2}-\bar{\delta} \phi_{1} & =-\lambda \phi_{0}+2 \pi \phi_{1}+(\rho-2 \epsilon) \phi_{2},
\end{aligned}
$$

where $\phi_{0}, \phi_{1}$ and $\phi_{2}$ are the Maxwell spinors, $\epsilon, \rho, \pi, \alpha, \mu, \gamma, \beta$ and $\tau$ are the spin coefficients to be found and the bar denotes complex conjugation. The null tetrad vectors for the metric (2.5) are defined by

$$
l^{a}=\left(\frac{1}{B}, 1,0,0\right)
$$




$$
\begin{aligned}
n^{a} & =\left(\frac{1}{2},-\frac{B}{2}, 0,0\right), \\
m^{a} & =\frac{1}{\sqrt{2}}\left(0,0, \frac{1}{r}, \frac{i}{r \sin \theta}\right) . \\
\bar{m}^{a} & =\frac{1}{\sqrt{2}}\left(0,0, \frac{1}{r}, \frac{-i}{r \sin \theta}\right)
\end{aligned}
$$

The directional derivatives in the Maxwell's equations are defined by $D=l^{a} \partial_{a}, \Delta=n^{a} \partial_{a}$ and $\delta=m^{a} \partial_{a}$. We define operators in the following way by assuming $\phi_{\alpha}=\phi_{\alpha}(r, \theta) e^{i(\omega t+m \varphi)}$ $(\alpha=0,1,2)$

$$
\begin{aligned}
& \mathbf{D}_{0}=D \\
& \mathbf{D}_{0}^{\dagger}=-\frac{2}{B} \Delta, \\
& \mathbf{L}_{0}^{\dagger}=\sqrt{2} r \delta \text { and } \mathbf{L}_{1}^{\dagger}=\mathbf{L}_{0}^{\dagger}+\frac{\cot \theta}{2} \\
& \mathbf{L}_{0}=\sqrt{2} r \bar{\delta} \text { and } \mathbf{L}_{1}=\mathbf{L}_{0}+\frac{\cot \theta}{2} .
\end{aligned}
$$

The non-zero spin coefficients are given in eqs. (2.27), (2.28). The Maxwell spinors are defined by [33]

$$
\begin{aligned}
\phi_{0} & =F_{13}=F_{\mu \nu} l^{\mu} m^{\nu} \\
\phi_{1} & =\frac{1}{2}\left(F_{12}+F_{43}\right)=\frac{1}{2} F_{\mu \nu}\left(l^{\mu} n^{\nu}+\bar{m}^{\mu} m^{\nu}\right), \\
\phi_{2} & =F_{42}=F_{\mu \nu} \bar{m}^{\mu} n^{\nu},
\end{aligned}
$$

where $F_{i j}(i, j=1,2,3,4)$ and $F_{\mu \nu}(\mu, \nu=0,1,2,3)$ are the components of the Maxwell tensor in the tetrad and tensor bases, respectively. Substituting eqs. (4.21)-(4.24) into the Maxwell's equations together with non-zero spin coefficients, the Maxwell equations become

$$
\begin{aligned}
\left(\mathbf{D}_{0}+\frac{2}{r}\right) \phi_{1}-\frac{1}{r \sqrt{2}} \mathbf{L}_{1} \phi_{0} & =0, \\
\left(\mathbf{D}_{0}+\frac{1}{r}\right) \phi_{2}-\frac{1}{r \sqrt{2}} \mathbf{L}_{0} \phi_{1} & =0, \\
\frac{B}{2}\left(\mathbf{D}_{0}^{\dagger}+\frac{B^{\prime}}{B}+\frac{1}{r}\right) \phi_{0}+\frac{1}{r \sqrt{2}} \mathbf{L}_{0}^{\dagger} \phi_{1} & =0, \\
\frac{B}{2}\left(\mathbf{D}_{0}^{\dagger}+\frac{2}{r}\right) \phi_{1}+\frac{1}{r \sqrt{2}} \mathbf{L}_{1}^{\dagger} \phi_{2} & =0 .
\end{aligned}
$$

The equations above will become more tractable if the variables are changed to

$$
\Phi_{0}=\phi_{0}, \quad \Phi_{1}=\sqrt{2} r \phi_{1}, \quad \Phi_{2}=2 r^{2} \phi_{2} .
$$

Then, we have

$$
\left(\mathbf{D}_{0}+\frac{1}{r}\right) \Phi_{1}-\mathbf{L}_{1} \Phi_{0}=0
$$




$$
\begin{array}{r}
\left(\mathbf{D}_{0}-\frac{1}{r}\right) \Phi_{2}-\mathbf{L}_{0} \Phi_{1}=0, \\
r^{2} B\left(\mathbf{D}_{0}^{\dagger}+\frac{B^{\prime}}{B}+\frac{1}{r}\right) \Phi_{0}+\mathbf{L}_{0}^{\dagger} \Phi_{1}=0, \\
r^{2} B\left(\mathbf{D}_{0}^{\dagger}+\frac{1}{r}\right) \Phi_{1}+\mathbf{L}_{1}^{\dagger} \Phi_{2}=0
\end{array}
$$

The commutativity of the operators $\mathbf{L}$ and $\mathbf{D}$ enables us to eliminate each $\Phi_{i}$ from above equations, and hence we have

$$
\begin{gathered}
{\left[\mathbf{L}_{0}^{\dagger} \mathbf{L}_{1}+r^{2} B\left(\mathbf{D}_{0}+\frac{B^{\prime}}{B}+\frac{3}{r}\right) \times\right.} \\
\left.\left(\mathbf{D}_{0}^{\dagger}+\frac{B^{\prime}}{B}+\frac{1}{r}\right)\right] \Phi_{0}(r, \theta)=0, \\
{\left[\mathbf{L}_{1} \mathbf{L}_{0}^{\dagger}+r^{2} B\left(\mathbf{D}_{0}^{\dagger}+\frac{B^{\prime}}{B}+\frac{1}{r}\right)\left(\mathbf{D}_{0}+\frac{1}{r}\right)\right] \Phi_{1}(r, \theta)=0 .} \\
{\left[\mathbf{L}_{0} \mathbf{L}_{1}^{\dagger}+r^{2} B\left(\mathbf{D}_{0}^{\dagger}+\frac{1}{r}\right)\left(\mathbf{D}_{0}-\frac{1}{r}\right)\right] \Phi_{2}(r, \theta)=0,}
\end{gathered}
$$

The variables $r$ and $\theta$ can be separated by assuming a separable solution in the form of

$$
\begin{aligned}
& \Phi_{0}(r, \theta)=R_{0}(r) \Theta_{0}(\theta), \\
& \Phi_{1}(r, \theta)=R_{1}(r) \Theta_{1}(\theta), \\
& \Phi_{2}(r, \theta)=R_{2}(r) \Theta_{2}(\theta) .
\end{aligned}
$$

The separation constants for eq. (4.37) and eq. (4.38) are the same, because $\mathbf{L}_{n}=-\mathbf{L}_{n}^{\dagger}(\pi-\theta)$, or, in other words, the operator $\mathbf{L}_{0}^{\dagger} \mathbf{L}_{1}$ acting on $\Theta_{0}(\theta)$ is the same as the operator $\mathbf{L}_{0} \mathbf{L}_{1}^{\dagger}$ acting on $\Theta_{2}(\theta)$ if we replace $\theta$ by $\pi-\theta$. However, for eq. (4.39) we will assume another separation constant. Furthermore, by defining $R_{0}(r)=\frac{f_{0}(r)}{r B(r)}, R_{1}(r)=\frac{f_{1}(r)}{r}$ and $R_{2}(r)=\frac{f_{2}(r)}{r}$, the radial equations can be written as

$$
\begin{array}{r}
f_{0}^{\prime \prime}(r)+\frac{2}{r} f_{0}^{\prime}(r)+\left[-i \omega\left(\frac{2}{r B}-\frac{B^{\prime}}{B^{2}}\right)+\frac{\omega^{2}}{B^{2}}-\frac{\epsilon^{2}}{r^{2} B}\right] f_{0}(r)=0, \\
f_{1}^{\prime \prime}(r)+\frac{B^{\prime}}{B} f_{1}^{\prime}(r)+\left[\frac{\omega^{2}}{B^{2}}-\frac{\eta^{2}}{r^{2} B}\right] f_{1}(r)=0, \\
f_{2}^{\prime \prime}(r)-\frac{2}{r} f_{2}^{\prime}(r)+\left[i \omega\left(\frac{2}{r B}-\frac{B^{\prime}}{B^{2}}\right)+\frac{\omega^{2}}{B^{2}}-\frac{\epsilon^{2}}{r^{2} B}\right] f_{2}(r)=0,
\end{array}
$$

where $\epsilon$ and $\eta$ are the separability constants and $\omega$ denotes the frequency of the photon wave.

The definition of the quantum singularity for Maxwell fields will be the same as for the Klein-Gordon fields. Here, since we have three equations governing the dynamics of the photon waves, the unique self-adjoint extension condition on the spatial part of the Maxwell operator should be examined for each of the three equations for all space. 


\subsubsection{For the case $r \rightarrow \infty$}

The corresponding metric is given in eq. (4.5). Hence, the radial parts of the Maxwell equations, (4.43), (4.44) and (4.45), become

$$
\begin{aligned}
& f_{0}^{\prime \prime}(r)+\frac{\omega\left(\omega-i \varphi_{0}\right)}{\left(\alpha-\psi_{0} r\right)^{2}} f_{0}(r)=0, \\
& f_{1}^{\prime \prime}(r)+\frac{\omega^{2}}{\left(\alpha-\psi_{0} r\right)^{2}} f_{1}(r)=0 \\
& f_{2}^{\prime \prime}(r)+\frac{\omega\left(\omega+i \varphi_{0}\right)}{\left(\alpha-\psi_{0} r\right)^{2}} f_{2}(r)=0,
\end{aligned}
$$

Thus, the solutions in the asymptotic case are

$$
\begin{aligned}
& f_{0}(r)=C_{1}\left(\alpha-\psi_{0} r\right)^{\frac{\varphi_{0}+i \omega}{\varphi_{0}}}+C_{2}\left(\alpha-\psi_{0} r\right)^{\frac{-i \omega}{\varphi_{0}}} \\
& f_{1}(r)=C_{3}\left(\alpha-\psi_{0} r\right)^{\gamma_{1}}+C_{4}\left(\alpha-\psi_{0} r\right)^{\gamma_{1}}, \\
& f_{2}(r)=C_{5}\left(\alpha-\psi_{0} r\right)^{\frac{\varphi_{0}-i \omega}{\varphi_{0}}}+C_{6}\left(\alpha-\psi_{0} r\right)^{\frac{i \omega}{\varphi_{0}}}
\end{aligned}
$$

in which $C_{i}$ are integration constants, $\gamma_{1}=\frac{\psi_{0}+\sqrt{\psi_{0}^{2}-4 \omega^{2}}}{2 \psi_{0}}$ and $\gamma_{2}=\frac{\psi_{0}-\sqrt{\psi_{0}^{2}-4 \omega^{2}}}{2 \psi_{0}}$. The square integrability condition at infinity is checked by calculating the squared norm of each solution $f_{i}$

$$
\left\|f_{i}\right\|^{2}=\int_{r}^{\infty} \frac{\left|f_{i}(r)\right|^{2} r^{2}}{\left(\alpha-\psi_{0} r\right)} d r . \quad i=0,1,2
$$

Calculations has revealed that the obtained solutions do not belong to the Hilbert space because $\left\|f_{i}\right\|^{2} \rightarrow \infty$.

\subsubsection{The case $\mathrm{r} \rightarrow 0$}

The metric near $r \rightarrow 0$ is given in eq. (4.9). Hence, the radial parts of the Maxwell equations (4.43), (4.44) and (4.45) for this case are given by

$$
\begin{aligned}
& f_{0}^{\prime \prime}(r)+\frac{2}{r} f_{0}^{\prime}(r)+\frac{a_{0}}{r} f_{0}(r)=0, \\
& f_{1}^{\prime \prime}(r)-\frac{1}{r} f_{1}^{\prime}(r)+\frac{b_{0}}{r} f_{0}(r)=0, \\
& f_{2}^{\prime \prime}(r)-\frac{2}{r} f_{2}^{\prime}(r)+\frac{a_{0}}{r} f_{0}(r)=0
\end{aligned}
$$

in which $a_{0}=\frac{\epsilon^{2}}{2 G M}, b_{0}=\frac{\eta^{2}}{2 G M}$ and solutions are obtained as,

$$
\begin{aligned}
& f_{0}(r)=\frac{C_{1}}{\sqrt{r}} J_{1}\left(2 \sqrt{a_{0} r}\right)+\frac{C_{2}}{\sqrt{r}} N_{1}\left(2 \sqrt{a_{0} r}\right), \\
& f_{1}(r)=C_{3} r J_{2}\left(2 \sqrt{b_{0} r}\right)+C_{4} r N_{2}\left(2 \sqrt{b_{0} r}\right), \\
& f_{2}(r)=C_{5} r^{3 / 2} J_{3}\left(2 \sqrt{a_{0} r}\right)+C_{6} r^{3 / 2} N_{3}\left(2 \sqrt{a_{0} r}\right),
\end{aligned}
$$

where $C_{i}$ are constants, $J_{i}$ and $N_{i}$ are Bessel and Neumann functions. The above solutions is checked for square integrability. Calculations have revealed that

$$
\left\|f_{i}\right\|^{2}=\int_{0}^{\text {constant }} \frac{\left|f_{i}(r)\right|^{2} r^{2}}{\left(\alpha-\frac{2 G M}{r}\right)} d r<\infty
$$


which indicates that the obtained solutions are square integrable. As a result, the spatial part of the Maxwell operator is not essentially self-adjoint and therefore, the occurrence of the timelike naked singularity in $f(R)$ gravity is quantum mechanically singular, if it is probed with photon waves.

\subsection{Dirac fields}

The Newman-Penrose formalism will also be used here to find the massless Dirac fields (fermions) propagating in the space of $f(R)$ global monopole spacetime. The Chandrasekhar-Dirac (CD) equations in the Newman-Penrose formalism are given by

$$
\begin{aligned}
(D+\epsilon-\rho) F_{1}+(\bar{\delta}+\pi-\alpha) F_{2} & =0, \\
(\Delta+\mu-\gamma) F_{2}+(\delta+\beta-\tau) F_{1} & =0, \\
(D+\bar{\epsilon}-\bar{\rho}) G_{2}-(\delta+\bar{\pi}-\bar{\alpha}) G_{1} & =0, \\
(\Delta+\bar{\mu}-\bar{\gamma}) G_{1}-(\bar{\delta}+\bar{\beta}-\bar{\tau}) G_{2} & =0,
\end{aligned}
$$

where $F_{1}, F_{2}, G_{1}$ and $G_{2}$ are the components of the wave function, $\epsilon, \rho, \pi, \alpha, \mu, \gamma, \beta$ and $\tau$ are the spin coefficients. The non-zero spin coefficients are given in eqs. (2.27), (2.28). The directional derivatives in the CD equations are the same as in the Maxwell equations. Substituting non-zero spin coefficients and the definitions of the operators given in eqs. (4.21)-(4.24) into the CD equations leads to

$$
\begin{array}{r}
\left(\mathbf{D}_{0}+\frac{1}{r}\right) F_{1}+\frac{1}{r \sqrt{2}} \mathbf{L}_{1} F_{2}=0, \\
-\frac{B}{2}\left(\mathbf{D}_{0}^{\dagger}+\frac{B^{\prime}}{2 B}+\frac{1}{r}\right) F_{2}+\frac{1}{r \sqrt{2}} \mathbf{L}_{1}^{\dagger} F_{1}=0, \\
\left(\mathbf{D}_{0}+\frac{1}{r}\right) G_{2}-\frac{1}{r \sqrt{2}} \mathbf{L}_{1}^{\dagger} G_{1}=0, \\
\frac{B}{2}\left(\mathbf{D}_{0}^{\dagger}+\frac{B^{\prime}}{2 B}+\frac{1}{r}\right) G_{1}+\frac{1}{r \sqrt{2}} \mathbf{L}_{1} G_{2}=0 .
\end{array}
$$

For the solution of the CD equations, we assume a separable solution in the form of

$$
\begin{aligned}
F_{1} & =f_{1}(r) Y_{1}(\theta) e^{i(k t+m \varphi)}, \\
F_{2} & =f_{2}(r) Y_{2}(\theta) e^{i(k t+m \varphi)}, \\
G_{1} & =g_{1}(r) Y_{3}(\theta) e^{i(k t+m \varphi)}, \\
G_{2} & =g_{2}(r) Y_{4}(\theta) e^{i(k t+m \varphi)},
\end{aligned}
$$

where $m$ is the azimuthal quantum number and $k$ is the frequency of the Dirac fields, which is assumed to be positive and real. Since $\left\{f_{1}, f_{2}, g_{1}, g_{2}\right\}$ and $\left\{Y_{1}, Y_{2}, Y_{3}, Y_{4}\right\}$ are functions of $r$ and $\theta$, respectively, by substituting eqs. (4.68)-(4.71) into eqs. (4.64)-(4.67) and applying the assumptions given by

$$
\begin{gathered}
f_{1}(r)=g_{2}(r) \quad \text { and } \quad f_{2}(r)=g_{1}(r), \\
Y_{1}(\theta)=Y_{3}(\theta) \quad \text { and } \quad Y_{2}(\theta)=Y_{4}(\theta),
\end{gathered}
$$


the Dirac equations transform into eqs. (4.74), (4.75) below. In order to solve the radial equations, the separation constant $\lambda$ should be defined. This is achieved by using the angular equations. In fact, it is already known from the literature that the separation constant can be expressed in terms of the spin-weighted spheroidal harmonics. The radial parts of the Dirac equations become

$$
\begin{aligned}
\left(\mathbf{D}_{0}+\frac{1}{r}\right) f_{1}(r) & =\frac{\lambda}{r \sqrt{2}} f_{2}(r), \\
\frac{B}{2}\left(\mathbf{D}_{0}^{\dagger}+\frac{B^{\prime}}{2 B}+\frac{1}{r}\right) f_{2}(r) & =\frac{\lambda}{r \sqrt{2}} f_{1}(r) .
\end{aligned}
$$

We further assume that

$$
\begin{aligned}
& f_{1}(r)=\frac{\Psi_{1}(r)}{r}, \\
& f_{2}(r)=\frac{\Psi_{2}(r)}{r},
\end{aligned}
$$

then eqs. (4.74), (4.75) transforms into,

$$
\begin{aligned}
\mathbf{D}_{0} \Psi_{1} & =\frac{\lambda}{r \sqrt{2}} \Psi_{2}, \\
\frac{B}{2}\left(\mathbf{D}_{0}^{\dagger}+\frac{B^{\prime}}{2 B}\right) \Psi_{2} & =\frac{\lambda}{r \sqrt{2}} \Psi_{1} .
\end{aligned}
$$

Note that $\sqrt{\frac{B}{2}} \mathbf{D}_{0}^{\dagger} \sqrt{\frac{B}{2}}=\mathbf{D}_{0}^{\dagger}+\frac{B^{\prime}}{2 B}+\frac{1}{r}$, and using this together with the new functions

$$
\begin{aligned}
& R_{1}(r)=\Psi_{1}(r), \\
& R_{2}(r)=\sqrt{\frac{B}{2}} \Psi_{2}(r),
\end{aligned}
$$

and defining the tortoise coordinate $r_{*}$ as

$$
\frac{d}{d r_{*}}=B \frac{d}{d r}
$$

Eqs. (4.78), (4.79) become

$$
\begin{aligned}
& \left(\frac{d}{d r_{*}}+i k\right) R_{1}=\frac{\sqrt{B} \lambda}{r} R_{2}, \\
& \left(\frac{d}{d r_{*}}-i k\right) R_{2}=\frac{\sqrt{B} \lambda}{r} R_{1},
\end{aligned}
$$

In order to write eqs. (4.83), (4.84) in a more compact form, we combine the solutions in the following way:

$$
\begin{aligned}
& Z_{+}=R_{1}+R_{2}, \\
& Z_{-}=R_{2}-R_{1} .
\end{aligned}
$$


After doing some calculations we end up with a pair of one-dimensional Schrödinger-like wave equations with effective potentials,

$$
\begin{aligned}
\left(\frac{d^{2}}{d r_{*}^{2}}+k^{2}\right) Z_{ \pm} & =V_{ \pm} Z_{ \pm} \\
V_{ \pm} & =\left[\frac{B \lambda^{2}}{r^{2}} \pm \lambda \frac{d}{d r_{*}}\left(\frac{\sqrt{B}}{r}\right)\right] .
\end{aligned}
$$

In analogy with eq. (3.2), the radial operator $A$ for the Dirac equations can be written as,

$$
A=-\frac{d^{2}}{d r_{*}^{2}}+V_{ \pm}
$$

If we write the above operator in terms of the usual coordinates $r$, by using eq. (4.82), we have

$$
A=-\frac{d^{2}}{d r^{2}}-\frac{B^{\prime}}{B} \frac{d}{d r}+\frac{\lambda}{B}\left[\frac{\lambda}{r^{2}} \pm \frac{d}{d r}\left(\frac{\sqrt{B}}{r}\right)\right],
$$

Our aim now is to show whether this radial part of the Dirac operator is essentially self-adjoint or not. This will be achieved by considering eq. (3.7) and counting the number of solutions that do not belong to Hilbert space. Hence, eq. (3.7) becomes

$$
\left(\frac{d^{2}}{d r^{2}}+\frac{B^{\prime}}{B} \frac{d}{d r}-\frac{\lambda}{B}\left[\frac{\lambda}{r^{2}} \pm \frac{d}{d r}\left(\frac{\sqrt{B}}{r}\right)\right] \mp i\right) \psi(r)=0 .
$$

\subsubsection{For the case $r \rightarrow \infty$}

For the asymptotic case, $r \rightarrow \infty$, the above equation transforms to

$$
\frac{d^{2} \psi(r)}{d r^{2}} \pm i \psi(r)=0
$$

whose solution is

$$
\psi_{ \pm}(r)=C_{1} \sin \left[\frac{(1 \pm i)}{\sqrt{2}} r\right]+C_{2} \cos \left[\frac{(1 \pm i)}{\sqrt{2}} r\right]
$$

The square integrability condition at infinity is checked by calculating the squared norm of each sign of solution $\psi_{ \pm}(r)$

$$
\left\|\psi_{ \pm}(r)\right\|^{2}=\int_{r}^{\infty} \frac{\left|\psi_{ \pm}(r)\right|^{2} r^{2}}{\left(\alpha-\psi_{0} r\right)} d r
$$

The outcome of the calculations showed that the obtained solutions are not belong to the Hilbert space because $\left\|\psi_{ \pm}(r)\right\|^{2} \rightarrow \infty$.

\subsubsection{For the case $\mathrm{r} \rightarrow 0$}

Near $r \rightarrow 0$, the approximate metric is given in eq. (4.9) and hence, eq. (4.91) for $r \rightarrow 0$ becomes

$$
\frac{d^{2} \psi(r)}{d r^{2}}+\frac{i \xi}{r^{3 / 2}} \psi(r)=0,
$$


in which $\xi=\frac{ \pm \lambda-2}{2 \sqrt{2 G M}}$, whose solution is given by

$$
\begin{aligned}
\psi(r)= & C_{1}\left\{-(1-i) \sqrt{2} r^{1 / 4} J_{1}(X)+4 \sqrt{r \xi} J_{0}(X)\right\} \\
& +C_{2}\left\{-(1-i) \sqrt{2} r^{1 / 4} N_{1}(X)+4 \sqrt{r \xi} N_{0}(X)\right\}
\end{aligned}
$$

where $J_{i}(X)$ and $N_{i}(X)$ are Bessel functions of the first and second kind, and $X=2(1+i) \sqrt{2 \xi} r^{1 / 4}$. Checking for the square integrability near $r \rightarrow 0$ has revealed that both solutions are square integrable.

Hence, the radial operator of the Dirac field fails to satisfy a unique self-adjoint extension condition for the entire space. As a result, the occurrence of the timelike naked singularity in the context of $f(R)$ global monopole remains singular from the quantum mechanical point of view, if it is probed with fermions.

\section{Probing the singularity around BV spacetime with Maxwell and Dirac fields}

In this section, we will extend the study of Pitelli and Letelier [19] for the BV spacetime in which the bosonic waves obeying the Klein-Gordon equation is used to probe the singularity to the spinor fields obeying the Maxwell and Dirac equations. Our motivation here is to check whether the spinorial waves can smooth out the singularity or not. The metric describing global monopole was obtained by BV and given by

$$
d s^{2}=d t^{2}-d r^{2}-a^{2} r^{2}\left(d \theta^{2}+\sin ^{2} \theta d \varphi^{2}\right) .
$$

The appropriate tetrads and the non zero spin coefficients are given by

$$
\begin{aligned}
l^{a} & =(1,1,0,0), \\
n^{a} & =\left(\frac{1}{2},-\frac{1}{2}, 0,0\right), \\
m^{a} & =\frac{1}{\sqrt{2}}\left(0,0, \frac{1}{a r}, \frac{i}{r a \sin \theta}\right) . \\
\bar{m}^{a} & =\frac{1}{\sqrt{2}}\left(0,0, \frac{1}{a r}, \frac{-i}{r a \sin \theta}\right) \\
\mu & =-\frac{1}{2 r}, \rho=-\frac{1}{r}, \beta=-\alpha=\frac{1}{2 \sqrt{2}} \frac{\cot \theta}{r a} .
\end{aligned}
$$

The non-vanishing tetrad fields are

$$
\Psi_{2}=-2 \Lambda=-\frac{2}{3} \phi_{11}=\frac{1}{6 r^{2}}\left(1-\frac{1}{a^{2}}\right)
$$

which vanish for $a= \pm 1$. 


\subsection{Maxwell fields}

Following the same steps of the previous section, the radial part of the Maxwell's equations (4.13)-(4.16) governing the photon waves are obtained as

$$
\begin{array}{r}
f_{0}^{\prime \prime}(r)+\left[\omega^{2}-\frac{2 i \omega}{r}-\frac{\epsilon^{2}}{r^{2} a^{2}}\right] f_{0}(r)=0, \\
f_{1}^{\prime \prime}(r)+\left[\omega^{2}-\frac{\eta^{2}}{r^{2} a^{2}}\right] f_{1}(r)=0, \\
f_{2}^{\prime \prime}(r)+\left[\omega^{2}+\frac{2 i \omega}{r}-\frac{\epsilon^{2}}{r^{2} a^{2}}\right] f_{2}(r)=0 .
\end{array}
$$

\subsubsection{For the case $r \rightarrow \infty$}

For the asymptotic case the Maxwell's equations reduces to

$$
f_{i}^{\prime \prime}(r)+\omega^{2} f_{i}(r)=0, \quad i=0,1,2
$$

whose solution is

$$
f_{i}(r)=C_{1} \sin (\omega r)+C_{2} \cos (\omega r), \quad i=0,1,2
$$

in which $C_{1}$ and $C_{2}$ are arbitrary constants. The square integrability condition at infinity is calculated by

$$
\left\|f_{i}(r)\right\|^{2}=\int_{r}^{\infty}\left|f_{i}(r)\right|^{2} r^{2} d r
$$

and it is found that the squared norm $\left\|f_{i}(r)\right\|^{2} \rightarrow \infty$. This result indicates that all the asymptotic solutions of the Maxwell's equation do not belong to the Hilbert space.

\subsubsection{For the case $\mathrm{r} \rightarrow 0$}

The Maxwell's equations near $r=0$ behaves as

$$
\begin{aligned}
& f_{i}^{\prime \prime}(r)-\frac{\epsilon^{2}}{r^{2} \alpha^{2}} f_{i}(r)=0, \quad i=0,2 \\
& f_{1}^{\prime \prime}(r)-\frac{\eta^{2}}{r^{2} \alpha^{2}} f_{1}(r)=0 .
\end{aligned}
$$

The solutions to these equations are obtained as

$$
f_{i}(r)=C_{3 i} r^{\gamma_{1}}+C_{4 i} r^{\gamma_{2}}, \quad i=0,2
$$

and

$$
f_{1}(r)=C_{5} r^{\gamma_{3}}+C_{6} r^{\gamma_{4}}
$$

where $C_{3 i}, C_{4 i}, C_{5}$ and $C_{6}$ are arbitrary constants. The exponents are given by

$$
\gamma_{1}=\frac{1}{2}\left(1+\sqrt{1+\frac{4 \epsilon^{2}}{a^{2}}}\right),
$$




$$
\begin{aligned}
& \gamma_{2}=\frac{1}{2}\left(1-\sqrt{1+\frac{4 \epsilon^{2}}{a^{2}}}\right), \\
& \gamma_{3}=\frac{1}{2}\left(1+\sqrt{1+\frac{4 \eta^{2}}{a^{2}}}\right), \\
& \gamma_{4}=\frac{1}{2}\left(1-\sqrt{1+\frac{4 \eta^{2}}{a^{2}}}\right) .
\end{aligned}
$$

The square integrability near $r=0$ is checked by calculating the squared norms of the obtained solutions by

$$
\left\|f_{i}\right\|^{2}=\int_{0}^{\text {constant }}\left|f_{i}(r)\right|^{2} r^{2} d r
$$

Our analysis has revealed that, if $C_{3 i}=C_{5}=0$ together with $\frac{\epsilon^{2}}{\alpha^{2}}>\frac{15}{4}$ and $\frac{\eta^{2}}{\alpha^{2}}>\frac{15}{4}$, the squared norms diverges. This result implies that the solutions for these specific modes do not belong to the Hilbert space.

Consequently, in contrast to the bosonic wave probe reported in [19], the classical singularity at $r=0$, for global monopole spacetime due to the $\mathrm{BV}$, remains quantum mechanically nonsingular with respect to the photonic wave probe that has spin 1 .

\subsection{Dirac fields}

The Chandrasekhar-Dirac equations given in eqs. (4.60)-(4.63) is solved by using the Newman-Penrose formalism for the ordinary global monopole metric (5.1). The same steps are followed as in section IV and hence, we end up with a pair of one-dimensional Schrödinger-like wave equations with effective potentials,

$$
\begin{aligned}
\left(\frac{d^{2}}{d r^{2}}+k^{2}\right) Z_{ \pm} & =V_{ \pm} Z_{ \pm} \\
V_{ \pm} & =\frac{\lambda^{\prime 2}}{r^{2}} \mp \frac{\lambda^{\prime}}{r^{2}}
\end{aligned}
$$

in which $\lambda^{\prime}=\frac{\lambda}{\alpha}$. Comparing with the equation (3.2), the radial operator $A$ for the Dirac equations can be written as

$$
A=-\frac{d^{2}}{d r^{2}}+V_{ \pm}
$$

As a requirement of the HM criterion, the radial Dirac operator $A$ should be examined whether it is essentially self-adjoint or not. We obtain this by considering eq. (3.7) and counting the number of solutions for each sign that do not belong to Hilbert space. Hence, we have

$$
\left(\frac{d^{2}}{d r^{2}}-\left[\frac{\lambda^{\prime 2}}{r^{2}} \mp \frac{\lambda^{\prime}}{r^{2}}\right] \mp i\right) \psi(r)=0 .
$$

\subsubsection{For the case $r \rightarrow \infty$}

The behavior of the eq. (5.26), as $r \rightarrow \infty$ is

$$
\left(\frac{d^{2}}{d r^{2}} \mp i\right) \psi(r)=0,
$$


whose solutions for each sign is

$$
\psi_{ \pm}(r)=C_{1 \pm} \sin \left(\frac{1}{\sqrt{2}}(1 \pm i) r\right)+C_{2 \pm} \cos \left(\frac{1}{\sqrt{2}}(1 \pm i) r\right)
$$

in which $C_{1 \pm}$ and $C_{2 \pm}$ are arbitrary integration constants for each sign of solution. Our calculations has shown that, the squared norms for each sign of solutions diverges, that is

$$
\left\|\psi_{ \pm}(r)\right\|^{2}=\int_{r}^{\infty}\left|\psi_{ \pm}(r)\right|^{2} r^{2} d r \rightarrow \infty
$$

indicating that the solutions at infinity do not belong to the Hilbert space.

\subsubsection{For the case $\mathrm{r} \rightarrow 0$}

The behavior of the eq. (5.26), near $r=0$ is,

$$
\left(\frac{d^{2}}{d r^{2}}-\frac{\lambda^{\prime}}{r^{2}}\left[\lambda^{\prime} \mp 1\right]\right) \psi(r)=0 .
$$

The solution is

$$
\psi(r)=C_{3} r^{\tau_{1}}+C_{4} r^{\tau_{2}},
$$

in which $C_{3}$ and $C_{4}$ are arbitrary constants. The exponents are given by

$$
\begin{aligned}
& \tau_{1}=\frac{1}{2}\left(1+\sqrt{1+4 \lambda^{\prime}\left(\lambda^{\prime} \pm 1\right)}\right), \\
& \tau_{1}=\frac{1}{2}\left(1-\sqrt{1+4 \lambda^{\prime}\left(\lambda^{\prime} \pm 1\right)}\right) .
\end{aligned}
$$

The obtained solution fails to be square integrable, if $C_{3}=0$ and $\lambda^{\prime}\left(\lambda^{\prime} \pm 1\right)>\frac{15}{4}$. Hence, solutions for these modes do not belong to the Hilbert space. As a result, the classical singularity at $r=0$, remains quantum mechanically nonsingular, if it is probed with fermions whose spin structure is $1 / 2$.

\section{Analysis with Gubser's singularity conjecture}

In this section, Gubser's [29] singularity conjecture will be used to analyse the timelike naked singularity in the $f(R)$ global monopole spacetime. It should be noted that this conjecture is based on investigating the behavior of the scalar potential $V(\vec{\varphi})$ on shell. Hence, the Gubser's singularity conjecture, investigates the singularity from a geometric point of view. Apparently different but structurally equivalent singularity criteria are proposed by Kim in [35], in which $D+1$ dimensional geometry with $D$ Poincare invariant spacetime is considered in the following form,

$$
d s^{2}=a(y)^{2} \eta_{\mu \nu} d x^{\mu} d x^{\nu}+d y^{2} .
$$

It is argued that, if the integral of the on-shell Lagrangian density over the finite range of $y$, whose least upper bound is $y=y_{c}$, is finite, the singularity at $y=y_{c}$ is physically admissible. 


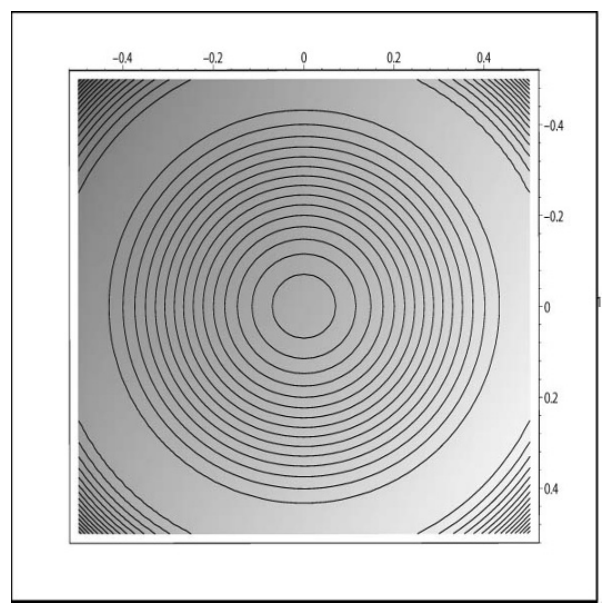

Figure 4. Contour plot of $V\left(\phi^{a}\right)$ with respect to $\phi^{1}$ and $\phi^{2}$ when $\left(\phi^{3}\right)^{2}-\eta^{2}=-\frac{1}{4}$ and $\lambda=1$. A local / absolute maximum is observed at $\left(\phi^{1}\right)^{2}+\left(\phi^{2}\right)^{2}=\frac{1}{4}$ and therefore the potential is bounded from above. From the Gubser's conjecture the large scalar curvature in this spacetime is allowed.

The 4-dimensional global monopole spacetime is governed by the triplet scalar field coupled with gravity. Therefore, we believe that this conjecture is applicable in this theory too. We consider the simplest case in which the action is given by

$$
I=\int d^{4} x \sqrt{-g}\left(R+\frac{1}{2} \partial_{\mu} \phi^{a} \partial^{\mu} \phi^{a}+V\left(\phi^{a}\right)\right)
$$

where $V\left(\phi^{a}\right)=-\frac{1}{4} \lambda\left(\phi^{a} \phi^{a}-\eta^{2}\right)^{2}$ and $a=1,2,3[30]$. It is clear that $V\left(\phi^{a}\right)$ has a local maximum at $\phi^{a} \phi^{a}=\eta^{2}$. Figure 4 shows a contour plot of $V\left(\phi^{a}\right)$ with respect to $\phi^{1}$ and $\phi^{2}$ while $\left(\phi^{3}\right)^{2}-\eta^{2}=-\frac{1}{4}$ and $\lambda=1$. The corresponding superpotential [32] $W\left(\phi^{a}\right)=-\frac{\sqrt{3}}{2}\left(\left(\phi^{1}\right)^{2}+\left(\phi^{2}\right)^{2}+\frac{1}{4}\right)$ is also plotted in figure 5. Based on these figures one concludes that according to the Gubser's conjecture the singularity of this spacetime is admissible i.e. a 'good' one.

\section{Conclusion}

In this paper, the formation of the timelike naked singularity in $f(R)$ global monopole spacetime is investigated within the framework of quantum mechanics. The timelike naked singularity developed at $r=0$, is probed with the quantum fields obeying the Klein-Gordon, Maxwell and Dirac equations. Our investigation is based on the criterion proposed by HM that incorporates the essential self-adjointness of the spatial part of the wave operator $A$ in the natural Hilbert space of quantum mechanics which is a linear function space with square integrability.

In this paper, the spinorial field generalization of the quantum singularity analysis of the BV spacetime reported in [19] is also studied. In order to show the influence of the modified theory on the singularity structure, we compare the results of the standard general relativity and $f(R)$ theory. 


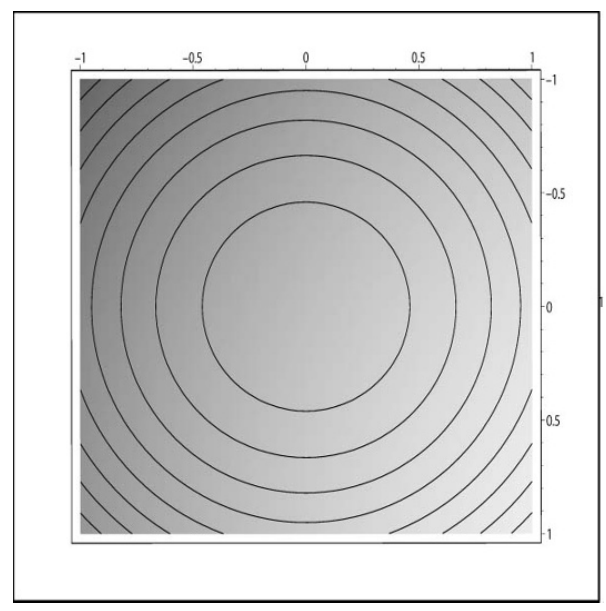

Figure 5. Contour plot of $W\left(\phi^{a}\right)$ with respect to $\phi^{1}$ and $\phi^{2}$ when $\left(\phi^{3}\right)^{2}-\eta^{2}=-\frac{1}{4}$ and $\lambda=1$. A local / absolute maximum is observed at $\left(\phi^{1}\right)=\left(\phi^{2}\right)=0$ and therefore the superpotential is bounded from above.

We showed with explicit calculations that the naked singularity at $r=0$, for the $f(R)$ global monopole spacetime, remains quantum mechanically singular when it is probed with quantum fields having different spin structures obeying Klein-Gordon, Maxwell and Dirac equations. It should be noted that in the analysis of $f(R)$ global monopole; although the mass term vanishes for large values of $r$ as in the case of BV spacetime, unlike the case in BV, the mass term becomes effective for $r \rightarrow 0$. Because of this nature, the singularity at $r=0$ becomes very strong in such a way that irrespective of the spin structure of the fields used to probe the singularity, the $f(R)$ global monopole spacetime remains quantum mechanically singular.

An interesting result is obtained for the spinorial wave probe generalization of the BV spacetime considered in [19]. We proved that for specific modes of solutions of the Maxwell and Dirac equations, the singularity at $r=0$ is smoothed out. The main reason of this result, seems to be the absence of the mass term. In addition, briefly we considered the geometrical approach of Gubser [32] to singularities in the present problem of cosmic string singularity.

It will be interesting for future research to extend the quantum singularity analysis in other $f(R)$ gravity models. Furthermore, it will be a great achievement if the criterion proposed by HM is extended to stationary metrics. Although the preliminary work in this direction is considered in [35], the formulation has not been fully completed.

Open Access. This article is distributed under the terms of the Creative Commons Attribution License (CC-BY 4.0), which permits any use, distribution and reproduction in any medium, provided the original author(s) and source are credited.

\section{References}

[1] G. Ellis and B. Schmidt, Singular space-times, Gen. Rel. Grav. 8 (1977) 915 [inSPIRE]. 
[2] P. Bell and P. Szekeres, Interacting electromagnetic shock waves in general relativity, Gen. Rel. Grav. 5 (1974) 275 [InSPIRE].

[3] G.T. Horowitz, Spacetime in string theory, New J. Phys. 7 (2005) 201 [gr-qc/0410049] [INSPIRE].

[4] M. Natsuume, The singularity problem in string theory, gr-qc/0108059 [INSPIRE].

[5] A. Ashtekar, Singularity resolution in loop quantum cosmology: a brief overview, J. Phys. Conf. Ser. 189 (2009) 012003 [arXiv: 0812.4703] [INSPIRE].

[6] J. Polchinski, String theory, Cambridge University Press, Cambridge U.K. (1998).

[7] A. Giveon, B. Kol, A. Ori and A. Sever, On the resolution of the timelike singularities in Reissner-Nordström and negative mass Schwarzschild, JHEP 08 (2004) 014 [hep-th/0401209] [INSPIRE].

[8] R.M. Wald, Dynamics in nonglobally hyperbolic, static space-times, J. Math. Phys. 21 (1980) 2082.

[9] G.T. Horowitz and D. Marolf, Quantum probes of space-time singularities, Phys. Rev. D 52 (1995) 5670 [gr-qc/9504028] [INSPIRE].

[10] A. Ishibashi and A. Hosoya, Who's afraid of naked singularities? Probing timelike singularities with finite energy waves, Phys. Rev. D 60 (1999) 104028 [gr-qc/9907009] [INSPIRE].

[11] D. Konkowski and T. Helliwell, Quantum singularity of quasiregular space-times, Gen. Rel. Grav. 33 (2001) 1131 [InSPIRE].

[12] T. Helliwell, D. Konkowski and V. Arndt, Quantum singularity in quasiregular space-times, as indicated by Klein-Gordon, Maxwell and Dirac fields, Gen. Rel. Grav. 35 (2003) 79 [INSPIRE].

[13] D. Konkowski, T. Helliwell and C. Wieland, Quantum singularity of Levi-Civita space-times, Class. Quant. Grav. 21 (2004) 265 [gr-qc/0401009] [INSPIRE].

[14] D.A. Konkowski, C. Reese, T.M. Helliwell and C. Wieland, Classical and quantum singularities of Levi-Civita spacetimes with and without a cosmological constant, in Procedings of the Workshop on the Dynamics and Thermodynamics of Black holes and Naked Singularities, L. Fatibene, M. Francaviglia, R. Giambo and G. Megli eds., Milan Italy (2004) [Conf. Proc. C 0405132 (2004) 247] [gr-qc/0410114] [InSPIRE].

[15] D. Konkowski and T. Helliwell, Quantum singularities in static and conformally static space-times, Int. J. Mod. Phys. A 26 (2011) 3878 [Int. J. Mod. Phys. Conf. Ser. 3 (2011) 364] [arXiv:1112.5488] [INSPIRE].

[16] T. Helliwell and D. Konkowski, Quantum singularities in spherically symmetric, conformally static spacetimes, Phys. Rev. D 87 (2013) 104041 [arXiv:1302.3970] [INSPIRE].

[17] P.M. Pitelli and P.S. Letelier, Quantum singularities in spacetimes with spherical and cylindrical topological defects, J. Math. Phys. 48 (2007) 092501 [arXiv:0708.2052] [INSPIRE].

[18] J.P.M. Pitelli and P.S. Letelier, Quantum singularities in the BTZ spacetime, Phys. Rev. D 77 (2008) 124030 [arXiv:0805.3926] [INSPIRE].

[19] J.P.M. Pitelli and P.S. Letelier, Quantum singularities around a global monopole, Phys. Rev. D 80 (2009) 104035 [arXiv:0911.2626] [INSPIRE]. 
[20] P.S. Letelier and J.P.M. Pitelli, n-dimensional FLRW quantum cosmology, Phys. Rev. D 82 (2010) 104046 [arXiv:1010.3054] [INSPIRE].

[21] O. Unver and O. Gurtug, Quantum singularities in $(2+1)$ dimensional matter coupled black hole spacetimes, Phys. Rev. D 82 (2010) 084016 [arXiv: 1004.2572] [INSPIRE].

[22] S.H. Mazharimousavi, O. Gurtug and M. Halilsoy, Generating static, spherically symmetric black-holes in third order Lovelock gravity, Int. J. Mod. Phys. D 18 (2009) 2061 [arXiv:0809.3649] [INSPIRE].

[23] S.H. Mazharimousavi, M. Halilsoy, I. Sakalli and O. Gurtug, Dilatonic interpolation between Reissner-Nordström and Bertotti-Robinson spacetimes with physical consequences, Class. Quant. Grav. 27 (2010) 105005 [arXiv:0908.3113] [INSPIRE].

[24] S.H. Mazharimousavi, O. Gurtug, M. Halilsoy and O. Unver, $2+1$ dimensional magnetically charged solutions in Einstein-power-Maxwell theory, Phys. Rev. D 84 (2011) 124021 [arXiv:1103.5646] [INSPIRE].

[25] T. Tahamtan and O. Gurtug, Quantum singularities in a model of $f(R)$ gravity, Eur. Phys. J. C 72 (2012) 2091 [arXiv:1205.5125] [INSPIRE].

[26] T. Carames, E. Bezerra de Mello and M. Guimaraes, Gravitational field of a global monopole in a modified gravity, Int. J. Mod. Phys. Conf. Ser. 3 (2011) 446 [arXiv:1106.4033] [INSPIRE].

[27] M. Barriola and A. Vilenkin, Gravitational field of a global monopole, Phys. Rev. Lett. 63 (1989) 341 [INSPIRE].

[28] J. Man and H. Cheng, The thermodynamic quantities of a black hole with an $f(R)$ global monopole, Phys. Rev. D 87 (2013) 044002 [arXiv:1301.2739] [INSPIRE].

[29] S.S. Gubser, Curvature singularities: the good, the bad and the naked, Adv. Theor. Math. Phys. 4 (2000) 679 [hep-th/0002160] [INSPIRE].

[30] M. Reed and B. Simon, Functional analysis, Academic Press, New York U.S.A. (1980).

[31] M. Reed and B. Simon, Fourier analysis and self-adjointness, Academic Press, New York U.S.A. (1975).

[32] R.D. Richtmyer, Principles of advanced mathematical physics, Springer, New York U.S.A. (1978).

[33] S. Chandrasekhar, The mathematical theory of black holes, Oxford University Press, Oxford U.K. (1992).

[34] I. Seggev, Dynamics in stationary, nonglobally hyperbolic space-times, Class. Quant. Grav. 21 (2004) 2651 [gr-qc/0310016] [INSPIRE].

[35] H.D. Kim, A criterion for admissible singularities in brane world, Phys. Rev. D 63 (2001) 124001 [hep-th/0012091] [INSPIRE]. 